\title{
Schmerzen nach inverser Schulterprothese: Skapulafraktur auschließen
}

Eine inverse Schulterprothese (Grammontprothese) kommt in erster Linie für Patienten infrage, bei denen eine Ruptur der Rotatorenmanschette, eventuell kombiniert mit einer Omarthrose, vorliegt oder bei denen eine konventionelle Arthroplastik zu einem unbefriedigenden Ergebnis geführt hat. Das inverse Verfahren entlastet die Rotatorenmanschette und kann zu einer besseren Beweglichkeit und Schmerzreduktion im Schultergelenk führen, birgt jedoch ein gewisses Risiko für Skapulafrakturen.

n einer retrospektiven Auswertung der Daten von 400 Patienten mit inverser Schulterprothese fanden US-amerikanische Autoren in 5,5\% der Fälle ipsilaterale Frakturen der Skapula. Die Forscher klassifizierten die Frakturen (Typ I-III) und verfolgten die jeweilige Therapie sowie den weiteren Verlauf über insgesamt viereinhalb Jahre.

Als Typ-I-Frakturen zählten kleinere Abrissfrakturen des anterioren Akromions nahe dem bzw. im Ansatz des Ligamentum coracoacromiale, die sich während der OP ereignet hatten und die auf Röntgenaufnahmen gut sichtbar waren. Frakturen vom Typ II betrafen das anteriore Akromion direkt hinter dem Akromioklavikulargelenk (AC-Gelenk); sie traten erst im Laufe des Follow-up nach dem Gelenkersatz auf. Letzteres galt auch für Typ-III-Frakturen, worunter die Autoren Frakturen des posterioren Akromions oder der Spina scapulae zusammenfassten.

Die acht Patienten mit Typ-I-Frakturen $(2,0 \%)$ konnten erfolgreich konservativ behandelt werden. Mit einer Ausnahme benötigten alle Betroffenen Steroidinjektionen zur Linderung von Schmerzen im AC-Gelenk.

Bei den zehn Patienten mit Typ-IIFrakturen $(2,5 \%)$ scheinen arthrotische Veränderungen und ein steifes AC-Gelenk mit Wiedererlangen der glenohumeralen Beweglichkeit nach der OP zu einer Dauerbelastung auf das Akromion geführt zu haben. Dies könnte das Risiko für eine Stressfraktur erhöhen. Die Patienten hatten nach der Implantation der inversen Prothese punktuell Schmerzen über dem anterioren Akromion entwickelt. Bei sieben Betroffenen wurde eine akromiale Stressfraktur nachgewiesen, in zwei Fällen war hierzu eine CT erforderlich. Sofern eine operative Versorgung vorgenommen wurde, konnte diese den Schmerz beseitigen. Die zunächst stabilen Typ-II-Frakturen, die man nur beobachtet hatte, waren alle im Laufe von zehn Monaten letztlich doch disloziert.

Die vier postoperativen Typ-III-Frakturen waren alle von vornherein disloziert; die Patienten erhielten eine offene Reposition mit interner Fixation (ORIF), was sie schmerzfrei werden ließ.

Schlussfolgerung: Die Typ-I-Skapulafraktur nach inverser Schulterendoprothese lässt sich erfolgreich konservativ behandeln. Bei Patienten mit mutmaßlicher Typ-II-Fraktur, die nach dem Eingriff einen punktuellen Schmerz über dem Akromion entwickeln, wird zur Abklärung einer Stressfraktur eine CT empfohlen. Bei Stabilität kann eine alleinige AC-Gelenk-Resektion ausreichen. Erscheint der Bruch instabil, sollte eine ORIF hinzukommen. Die Autoren empfehlen die ORIF auch bei allen Typ-IIIFrakturen.

Dr. Elke Oberhofer

Kommentar: Die retrospektive Untersuchung von Crosby, Hamilton und Twiss beleuchtet einen bisher wenig beachteten Aspekt der Schulterendoprothetik: Das Auftreten von Skapula-Frakturen als Folge der Implantation einer inversen Schulterprothese.

In einer retrospektiven Auswertung von 400 Patienten nach Implantation einer inversen Schulterprothese fanden sich in 5,5\% ipsilaterale Skapulafrakturen, wobei eine Klassifikation in drei Frakturtypen vorgenommen wurde.

Typ I-Frakturen (kleine Abrissfrakturen des anterioren Akromiums nahe dem bzw.

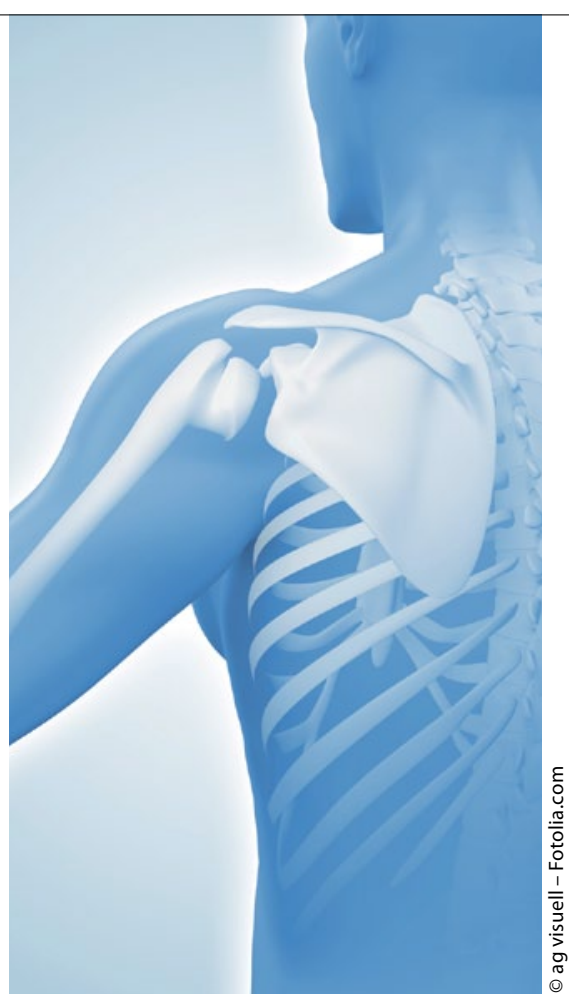

Skapulafrakturen nach inverser Schulterprothese lassen sich in drei Typen unterteilen.

im Ansatz des Ligamentum coracoacromiale) konnten erfolgreich konservativ behandelt werden.

Frakturen vom Typ II (Fraktur des anterioren Akromions direkt hinter dem ACGelenk) und des Typs III (Frakturen des posterioren Akromioms oder der Spina scapulae) bedürfen einer operativen $\mathrm{Be}$ handlung.

Diese Ergebnisse sind nicht nur für den Schulteroperateur, sondern auch für den Nachbehandler von Bedeutung. Klagt ein Patient nach Implantation einer inversen Schulterprothese über Schulterschmerzen, kann mit Röntgen und gegebenenfalls $C T$ nach einer Fraktur des Akromioms oder der Spina scapulae gefahndet werden.

Crosby LA et al. Scapula Fractures After Reverse Total Shoulder Arthroplasty: Classification and Treatment. Clin Orthop Relat Res 2011;469:2544-2549

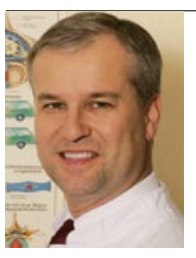

Prof. Dr. med. Klaus M. Peters Chefarzt der Orthopädie und Osteologie Rhein-Sieg-Klinik Höhenstraße 30 51588 Nümbrecht 